\title{
Algorithm for Leader Node Selection in Vehicular Adhoc Networks at Road Intersections
}

\author{
Sreyash Ratna Tripathi, Shreya Mahabala Alva and Debasis Das ${ }^{+}$ \\ Department of Computer Science and Engineering, NIIT University,Neemrana, Rajasthan, India
}

\begin{abstract}
With advancement in the field of vehicle automation and wireless communication, Vehicular Ad Hoc network (VANET) based solutions are emerging as the answer to the dire problem of road traffic management. As dedicated short-range communications (DSRC) devices, vehicles can be organized in a peer-to-peer network to manage their movement for a smoother traffic flow. Our paper targets traffic management at road intersections. Our approach calls for the selection of a leader node in every lane so that they may mutually decide a safe and efficient order of crossing the intersection. Generally leader node selection algorithms have a computational complexity of $\mathrm{O}(\mathrm{n} \log (\mathrm{n}))$. Our proposed algorithm uses geographic routing coupled with sequential flow of communication to select the leader node with a computational complexity of $\mathrm{O}(\mathrm{n})$ where $\mathrm{n}$ is the number of vehicles present in a lane at Road Intersection.
\end{abstract}

Keywords: node selection algorithm, leader node, king node, intelligent transportation system, road intersection, traffic management, cooperative intersection management, cooperative vehicle intersection control, vehicular adhoc network.

\section{Introduction}

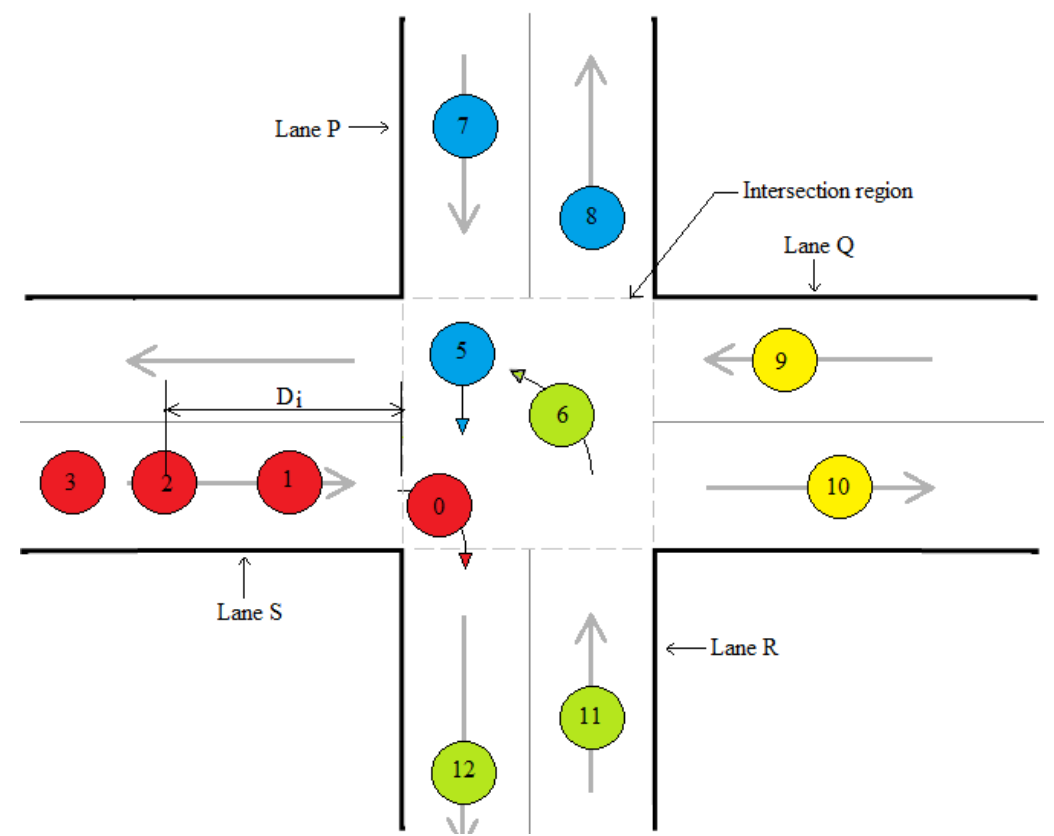

Fig. 1: A typical four-way bi-lane road intersection.

The world economy loses billions to road traffic congestion, associated delays and fuel wastage. With advancement in Vehicle-to-Vehicle(V2V)/Vehicle-to-Infrastructure(V2I) communication, in vehicle sensors

+ Corresponding author. Tel.: +91 7790844892;

E-mail address: Debasis.Das@niituniversity.in. 
and computer-based control, traffic management systems must evolve to harness the power of these technologies. Road Side Units (RSUs) can serve as the infrastructural support to co-ordinate vehicular movement however cost of installation and maintenance poses as a major drawback.

This paper proposes a new approach of selecting the leader node on any lane which is leading up to an intersection using only $\mathrm{V} 2 \mathrm{~V}$ communication. We have chosen geographic routing [9] (also known as position based routing) for cooperative intersection management. The leader node that will head communication for a set of vehicles. The leader nodes will communicate among themselves to decide their order of crossing. Global Positioning System (GPS) gives the location co-ordinates of each node. Each node receives this information pertaining its neighboring nodes. In every lane, the nodes that are closest to the intersection are allocated the rank 1 and chosen to be the leader nodes. Each node will deduce its own rank in the lane of vehicles based on the rank of the node that is directly ahead of it and thus closer to the intersection. Every time a node leaves its lane and enters the intersection the node that is right behind will assume rank 1 and will be communicating with the next set of designated leader nodes chosen from the other lanes.

\section{Related Work}

In Reference [1] J. Lee et al. designed a Cooperative Vehicle Intersection Control (CVIC) system for the Connected Vehicles environment. It relied on V2I and V2V communication for effective intersection management without a traffic signal, for fully automated vehicles. It sought to resolve all conflicting approaches with overlapping trajectories at the intersection and to chart out safe courses for all the vehicles. In Reference [2] I. Zohdy et al. used the concept of Cooperative Adaptive Cruise Control (CACC) in conjunction with the Vehicle Dynamics Model to draw up the speed distribution for the vehicles. It employed controllers at the "smart" intersections which counseled the vehicle, at each time step, regarding the best course of action to be taken to mitigate collision and decrease the total delay. In Reference [4] F. Perronnet et al. extended sequence-based protocol, called 'Transparent Intersection Management', for cooperative intersection management through a high-level signalization system (that is green or red displayed by an onboard screen to the driver) in which a speed limitation is determined by the sequence and adhered to by the autonomous vehicle. In Reference [5] A. Darehshoorzadeh et al. proposed Heuristic Candidate selection algorithm based on Optimum delivery Probability (HU-COP), a heuristic and fast candidate selection algorithm (in Opportunistic Routing) based on the link delivery probability a candidate and another node. HU-COP finds candidates through links whose delivery probabilities are near optimum, outperforming ExOR. In Reference [6] Mengmeng Wang et al. Mengmeng Wang et al. explored the issues of routing that are particular to urban area VANETs. They evaluated the performance of the existing routing protocols including GPCR, GyTAR, STAR, and TDR in city scenarios and also put forward guidelines for designing VANETs routing protocols. In Reference [7] Xiangjun Qian et al. adopted a priority-based coordination framework to partition the problem into a priority assignment problem and a vehicle control problem under the assigned (fixed) priorities. In Reference [8] Acarman T. et al. presented a wireless access protocol that routes the data packet towards a vehicular node in a neighbor intersection zone using navigation map data and routing information. In their algorithm, routing decision is chiefly made at the intersection zones.

\section{Objective}

In this paper, our focus is on road intersections, and our approach involves selecting leader nodes for regulatory purposes.

The main objectives of our proposed approach are given below:

- Communication between candidate nodes will decide the vehicles' order and speed of crossing the intersection.

- To improve upon existing methods for finding a leader node by using vehicle to vehicle (V2V) communication to establish precedence among the vehicles in the intersection.

\section{Leader Node Selection in VANET at Road Intersections}




\subsection{Preliminaries and Data Structures}

In this section, we will introduce the basic terminologies and assumptions which are used in the proposed algorithm.

- GPS: Global Positioning System. It is a satellite based navigation system that will provide the location and time information for the vehicle.

- $T_{c}$ : It represents current time. \%timer starts when the node enters the network

- $T_{i}$ : It represents time taken by the vehicle to reach the intersection.

- $T_{d}$ : It represents a short waiting time.

- $T_{m}$ : Time of transmission of a message.

- $D_{i}$ : It is the distance of the vehicle from the Intersection. The first value of the $D_{i}$ is taken from the GPS. After that, $\mathrm{D}_{\mathrm{i}}$ is maintained as per the formula:

$D_{i}=D_{G P S}-S_{\text {vehicle }} * T_{c}-T_{\text {rec }}$ here, $T_{\text {rec }}$ is the time when the GPS Data was received and $D_{G P S}$ is the distance from intersection received from GPS.

- $D_{r}$ : It is the direction of the moving vehicle.

- $I_{\text {packet }}$ : It is an information packet broadcasted by the vehicle containing $V_{i d}, D_{i}, D_{r}$ and $T_{m}$.

- Session-Request: It is a message that requests for a session from another node to communicate further. It contains $\mathrm{V}_{\text {id }}$, Request Message, $D_{i}$ and $T_{m}$.

- Request-Accepted: It is a message that contains $V_{i d}, D_{r}, D_{i}$ of the vehicle and $T_{m}$.

- Interrupt: It is a signal generated shows that some error was found while executing the algorithm.

- Data-Packet: It is the message transfer packet containing the $V_{i d}$ of the requesting vehicle, $R_{m}$ the modified rank, current speed of the broadcasting vehicle and $T_{m}$.

\subsection{Algorithm 1: King Node Selection Algorithm}

Step 1: GPS notifies the vehicle entering a lane leading up to an intersection and sends the initial value of $D i$ to the vehicle.

Step 2: The vehicle starts listening for broadcasts of $I_{\text {packet }}$ from other nodes for a Wait-Time $T d$.

Step 3: If the vehicle hears a broadcast of an $I_{\text {packet }}$ during $T d$ then execute Algorithm 2 and returning from Algorithm 2 execute from Algorithm 1 Step 8.

Step 4: End If

Step 5: Assume Rank: 1 and start broadcasting sequence of $I_{\text {packet }}$ and continue to listen for any broadcasts of $I_{\text {packet }}$ from other nodes as well.

Step 6: If the vehicle hears a broadcast Then execute Algorithm 2.

Step 7: End If

Step 8: As soon as a Session-Request packet is received, pause the broadcasting sequence of the $I_{\text {packet }}$.

Step 9: Select the node which sent the first Session-Request packet.

Step 10: Broadcast a Request-Accepted packet for the selected node.

Step 11: Execute Algorithm 3

Step 12: If the broadcasting vehicle receives an Interrupt Then it shall resume it's broadcasting sequence which was paused in Step 8.

Step 13: Else the broadcasting vehicle starts Message Transfer in the Session by executing Algorithm 4 .

Step 14: End If

//The vehicle with Rank-1 is the King Node while the rest of the vehicles are the Follower Nodes.

Step 15: If (King Node enters the Intersection, i.e $D_{i}=0$, Then

Step 16: King node sends out a Good-Bye message to first follower.

Step 17: The first follower catches the Good-Bye message and updates its rank to 1 and becomes the next King Node.

Step 18: It then sends out a message to the next following node to upgrade its rank by 1 and accordingly the ranks get upgraded for all the vehicles in the lane. 
Step 19: End If

\subsection{Algorithm 2: Checking Broadcast Authenticity of Ipacket}

Step 1: If $D_{i}$ of the vehicle receiving the $I_{\text {packet }}>D_{i}$ present in the $I_{\text {packet }}$ broadcasted AND $D_{r}$ of the vehicle is same as $D_{r}$ present in the $I_{\text {packet }}$

Step 2: It means that a vehicle which is in the same lane and is closer to the intersection has broadcasted it's $I_{\text {packet }}$

Step 3: Drop the current assumptions and ranks

Step 4: Stop any broadcasting sequence

Step 5: Send a Session-Request packet to the broadcasting vehicle

Step 6: Else drop the received $I_{\text {packet }}$

Step 7: End If

\subsection{Algorithm 3: Checking Session Authenticity}

All the nodes which sent the Session-Request packet are expecting a reply. They will all hear any broadcast message.

Step 1: If Broadcasted Message reads Request-Accepted Then

Step 2: Check the $D_{r}$ of the receiving vehicle with the one given in the packet

Step 3: If $D_{r}$ of the receiving vehicle $=D_{r}$ given in Request-Accepted Packet Then

Step 4: Take the $D_{i}$ and time of message transmission $T_{m}$ from the Request-Accepted packet.

Step 5: Use the following equation to modify the given $D_{i}$ to $D_{m}$. $D_{m}=D_{i}-\left(S_{\text {vehicle }} *\left(T_{c}-T_{m}\right)\right)$

Step 6: where $T_{c}$ is the current time.

Step 7: This is done to compensate the change in transmitted $D_{i}$ in the message versus the current $D_{i}$ because of movement of vehicles.

Step 8: If $D_{m}>D_{i}$ of vehicle Then

Step 9: Generate an Interrupt

Step 10: Broadcast the Interrupt

Step 11: Exit Algorithm 3 and all nodes execute Algorithm 1 from Step 2

Step 12: Else All the non-selected nodes exit Algorithm 3 and execute Algorithm 1 from Step 2

Step 13: End If

Step 14: Else Ignore the current broadcast message.

Step 15: End If

Step 16: Ignore the current broadcast message.

Step 17: End If

\subsection{Algorithm 4: Message Transfer in a Session}

Step 1: The selected node with Request-Accepted packet replies with a Begin-Transmission packet to the broadcasting node.

Step 2: The broadcasting node, upon getting the Begin-Transmission, sends a Data-Packet to the vehicle with a modified rank $R_{m}=R_{\text {broadcasting-vehicle }}+1$ and the speed $S_{\text {broadcasting-vehicle }}$

Step 3: The vehicle receives this Data-Packet and changes it's rank to $R_{m}$ and changes it's speed to match $S_{\text {broadcasting-vehicle }}$

Step 4: Update $T_{\text {rec }}$ as $T_{\text {rec }}=T_{c}$ and $D_{G P S}=D_{i}$. This will be used to update $D_{i}$.

Step 5: The vehicle then replies with an End-Transmission packet to the broadcasting node and start its broadcasting sequence of $I_{\text {packet }}$ with the modified data. 
Step 6: Upon receiving this End-Transmission packet the broadcasting node stops broadcasting. However, it does not stop listening for future broadcasts of $I_{\text {packet }}$ from other vehicles.

Step 7: For the above condition, If the previously broadcasting vehicle hears a broadcast of an $I_{\text {packet }}$ Then execute Algorithm 1, from Step 6.

\section{Step 9: Else exit Algorithm 4}

\section{Step 10: End If}

The King node selection algorithm starts executing as soon as a node enters a lane leading up to an intersection. There are two possible scenarios for a node entering such a lane:

- Case 1: Node enters in an empty lane. i.e., no other vehicle is present in that lane.

- Case 2: Node enters in a busy lane. i.e., there are one or more vehicles already present in that lane.

The King Node selection algorithm accounts for both of these cases in the following manner (refer Fig. 2) Let $N_{l}$ enter an empty lane, as in Case 1. As $N_{l}$ enters the lane, it will sense the approaching intersection with the help of GPS intimation which will trigger Algorithm 1. $N_{l}$ starts listening for broadcast from any other node on the lane for $T_{d}$ time. After $T_{d}$ has elapsed, $N_{l}$ assumes rank 1 and starts broadcasting $I_{p a c k e t s}$ while continuing to listen for any incoming messages.

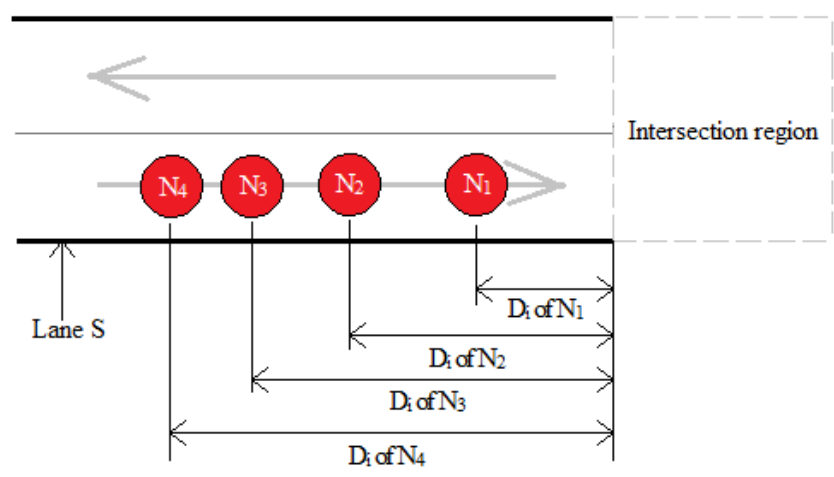

Fig. 2: Snapshot of Lane S.

Now suppose another node, $N_{2}$, enters the lane. $N_{2}$ will be entering a busy lane as in Case 2. $N_{2}$ will also start listening for a broadcast and will receive $I_{\text {packets }}$ from node N1 and after authentication of these $I_{\text {packets }}$, it will send a session request to $N_{l}$. However, if $N_{2}$ fails to listen to $N_{l}$ in the designated time, due to some reason, $N_{2}$ will also commence the broadcast of its $I_{\text {packets }}$ with the same assumptions as that of $N_{l}$ which creates a problem. To solve this problem, a protocol stated in Algorithm 2 is called. The protocol specifies that a node is supposed to accept and read only those broadcast messages which come from another node whose distance from intersection $D_{i}$ is lesser than their own $D_{i}$ and are traveling in the same direction. Therefore, even if $N_{2}$ starts broadcasting assuming it's rank to be 1, as soon as it receives a message from N1 which has a lesser Di than that of $N_{2}, N_{2}$ will stop it's current broadcast transmission.

For a case where multiple nodes, $N_{2}, N_{3}$ and $N_{4}$, enter the lane very close to each other, then all the nodes will hear $N_{l}$ broadcasting and send a session request to $N_{l}$. In such a case, $N_{l}$ will only entertain the session request of the node that that was sent first and respond by broadcasting a Request-Accepted packet with the senders $V_{i d}, D_{i}$ and $D_{r}$ to all the nodes. As two or more nodes may send their Session-Request packet at the same time, there is a possibility of a collision. Here, if nodes $N_{2}$ and $N_{3}$ have a broadcast storm and their Session-Request packets are lost then $N_{l}$ will only receive the session request from $N_{4}$. Now, Since $N_{2}$ and $N_{3}$ are waiting for a response, even they will read the Request-Accepted sent by $N_{l}$. If the $D_{i}$ mentioned in the Request-Accepted is more than that of $N_{2}$ or $N_{3}$, then they will generate and broadcast an Interrupt which will cause the session to terminate immediately and $N_{l}$ will resume it's broadcasting sequence.

When the authenticity of the session has been checked, the Communicating nodes start the message transfer. $N_{2}$, after receiving the Request-Accepted packet, will send a Begin-Transmission packet to $N_{l}$. $N_{l}$ will send the modified rank and speed to $N_{2} . N_{2}$ will modify it's rank and speed accordingly and send an EndTransmission packet to $N_{l}$. $N_{2}$ will then adjust the values of Trec and $D_{G P S}$ which is used to update $D_{i}$. When 
$N_{l}$ receives the End-Transmission packet, it will permanently stop the broadcasting stream of its $I_{\text {packet }}$ which was earlier paused, however, it will continue to listen for any other broadcast and $N 2$ will start its broadcast sequence of $I_{\text {packet }}$. When node $N_{l}$ enters the intersection, it will send a Good-Bye packet to the node with Rank 2 which is $N_{2}$. Upon receiving a Good-Bye message, $N_{2}$ decrements it's rank by 1 thereby becoming Rank 1 or the new King node. $N_{2}$ will further pass this message and so on and so forth till all the nodes in the lane adjust their ranks by 1 .

\section{Evaluation and Results}

In order to verify the performance our proposed algorithm, we developed a simulation environment using SUMO and NS2 which models the operation of a wireless network along a single road lane, connected to a road intersection. The simulator does not model medium contention or any radio propagation effects other than random packet loss based on a delivery ratio and assumes losses are not correlated with packet size. The simulated environment consists of nodes moving towards a fixed point (the intersection region) with fixed speed of $40 \mathrm{~km} / \mathrm{hr}$.

During our experiments, we first set number of vehicles in a lane, $n$, to $3,4,5$ and so on. Due to constraints of the simulator, we have limited the $n$ to 25 . The plot indicates that the number of messages transmitted to correctly assign ranks to each of the vehicles in a lane varies linearly as the number of vehicles in the given lane.

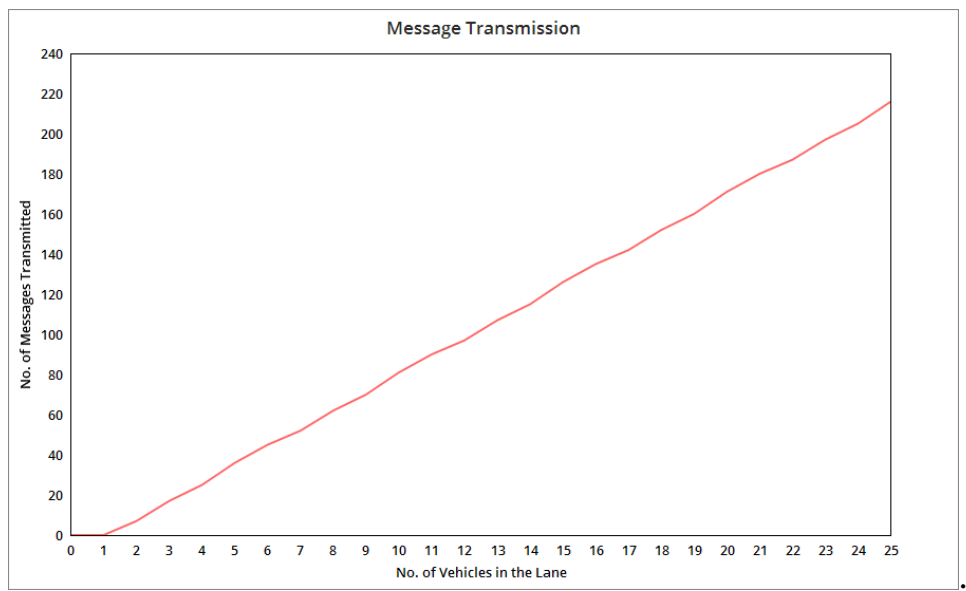

Fig. 2: Number of transmissions required to assign ranks to all nodes in a lane, averaged over number of vehicles in a single lane.

\section{Acknowledgements}

We'd like to thank our parents and our mentor for their constant support and guidance.

\section{Conclusion and Future Work}

Our approach, in this paper, was focused on V2V communication and position based routing, without relying on infrastructural support. In order to coordinate the movement of vehicles at a road intersection, the first step would be to selecting a leader node to function as the network coordinator. Existing leader selection algorithms have a computational complexity of $\mathrm{O}(\mathrm{n} \log n)$ or higher and in this paper, we have formulated an algorithm called as the King Node Selection algorithm with a complexity of $\mathrm{O}(\mathrm{n})$ that does not rely on infrastructural support.

In future, we shall extend our work by devising a priority based algorithm for a safe and efficient sequence of crossing to synchronize vehicle movement at intersections via communication between these chosen king nodes.

\section{References}


[1] J. Lee and B. Park, "Development and evaluation of a cooperative vehicle intersection control algorithm under the connected vehicles environment,” Intelligent Transportation Systems, IEEE Transactions on, vol. 13, pp. 81-90, March 2012.

[2] I. Zohdy, R. Kamalanathsharma, and H. Rakha, "Intersection management for autonomous vehicles using icacc," in Intelligent Transportation Systems (ITSC), 2012 15th International IEEE Conference on, pp. 1109-1114, Sept 2012.

[3] A. de La Fortelle, "Analysis of reservation algorithms for cooperative planning at intersections," in Intelligent Transportation Systems (ITSC), 2010 13th International IEEE Conference on, pp. 445-449, Sept 2010.

[4] F. Perronnet, A. Abbas-Turki, and A. El Moudni, "A sequenced-based protocol to manage autonomous vehicles at isolated intersections," in Intelligent Transportation Systems-(ITSC), 2013 16th International IEEE Conference on, pp. 1811-1816, IEEE, 2013.

[5] Darehshoorzadeh, A.; Boukerche, A., "An efficient heuristic candidate selection algorithm for Opportunistic Routing in wireless multihop networks," in Computers and Communication (ISCC), 2014 IEEE Symposium on , vol., no., pp.1-6, 23-26 June 2014

[6] Mengmeng Wang; Yankang Zhang; Changle Li; Xin Wang; Lina Zhu, "A survey on intersection-based routing protocols in city scenario of VANETs," in Connected Vehicles and Expo (ICCVE), 2014 International Conference on , vol., no., pp.821-826, 3-7 Nov. 2014

[7] Xiangjun Qian, Jean Gregoire, Fabien Moutarde, Arnaud De La Fortelle. Priority-based coordination of autonomous and legacy vehicles at intersection. 17th International IEEE Conference on Intelligent Transportation Systems, Oct 2014, Qingdao, China. <hal-01059090>

[8] Acarman, T.; Yaman, C.; Peksen, Y.; Peker, A.U., "Intersection Based Routing in Urban VANETs," in Intelligent Transportation Systems (ITSC), 2015 IEEE 18th International Conference on , vol., no., pp.1087-1092, 15-18 Sept. 2015.

[9] Debasis Das, Rajiv Misra and A. Raj “Approximating Geographic Routing using Coverage Tree Heuristics for Wireless Network”, Wireless Networks, Springer US, Vol. 21. No. 4, 2015, Pages 1109-1118. 